1 Médico de Servicio Social, Hospital General San Francisco, Juticalpa, Olancho.

2 Medico Interno Hospital Escuela Universitario, Tegusigalpa MDC, Francisco Morazan

$\overline{{ }^{3} \text { Estudiante de 6to año de Mé- }}$ dicina y Cirugia, Universidad Nacional Autonoma de Honduras.

${ }^{4}$ Doctora en Medicina Y cirugia, Universidad Nacional Autonoma de Honduras

Correspondencia a: ajovannaraquel@gmail.com

Palabras clave: Cardiovascular, Miocardio, infarto Agudo.

Keywords: Cardiovascular, Myocardio, acute myocardial infarction.

\title{
CARACTERIZACIÓN DE LOS FACTORES DE RIESGO CARDIOVASCULAR PARA INFARTO AGUDO DE MIOCARDIO EN POBLACIÓN GARÍFUNA
}

\author{
Characterization of cardiovascular risk factors for myocardial acute in- \\ farction in a Garifuna Population.
}
Luis Jose Pinto Garcia', Fernando Enrique Lobo Cerna², Jovanna Raquel Andrade Romero3, Elvia Maria Soriano ${ }^{4}$.

\section{RESUMEN}

Introducción. La enfermedad cardiovascular se caracteriza por una etiología multifactorial, siendo los factores de riesgos generalmente potenciados entre sí y con frecuencia asociados. Teniendo Se tuvo como objetivo, caracterizar los factores de riesgo cardiovascular para infarto agudo de miocardio en una población de raza Ggarífuna, tomando en cuenta factores de riesgo modificables y no modificables. Los resultados de este estudio, facilitarán la creación de programas de intervención para prevención de enfermedades cardiovasculares y la disminución de factores de riesgo en la población de Ciriboya y/o poblaciones similares.

Métodos. Se realizó una investigación cuantitativa de tipo observacional, descriptivo, de corte transversal. La muestra fue conformada por 139 personas, calculada con un nivel de confianza del 95\%, seleccionadas mediante un muestreo de tipo probabilístico aleatorio. Se utilizó la Encuesta de la Asociación Colombiana de Cardiología como instrumento de recolección de datos, la cual asigna puntaje a los factores de riesgo según su magnitud; se aplicó casa por casa a los miembros del hogar en el rango etario estudiado.

Resultados. Se encontró que $67 \%$ de los encuestados presenta riesgo cardiovascular; de las mujeres encuestadas, $64 \%$ presenta riesgo, mientras que $71 \%$ de los hombres presenta riesgo. Los factores de riesgo con mayor prevalencia son sobrepeso (58\%), antecedente familiar de hipertensión arterial (43\%), antecedente familiar de diabetes mellitus (35\%) e hipertensión arterial diagnosticada (32\%).

Conclusiones. En la población estudiada los hombres poseen mayor riesgo cardiovascular que las mujeres. Entre los factores de riesgo el más significativo fue sobrepeso.

\section{ABSTRACT}

Introduction. Cardiovascular disease is characterized by a multifactorial etiology, risk factors are aften associated and enhanced one another.Aiming to characterize the cardiovascular risk factors for acute heart attack in a population of African descents, taking modifiable and non-modifiable risk facts. The results will facilitate the creation of intervention programs to prevent cardiovascular diseases and decrease risk factors in the population of Ciriboya and similar populations.

Methods.A transversal descriptive research was conducted with quatitative approach. The sample was I39 people with confidence level of 95\%.A survay from The Colombian Association of Cardiology was used as data collection instrument, which assigns scores to the risk factors according to their magnitude; house-to-house applied to household members in the age range studied.

Results. It was found that $67 \%$ of the surveyed has risk; of women surveyed $64 \%$ is at risk, while $71 \%$ of men is at risk. The most prevalent risk factors are overweight (58\%), family history of hypertension (43\%), family history of diabetes mellitus (35\%) and diagnosed hypertension (32\%). Conclusions. In the studied population men have higher risk than women. Among the risk factors the most significant was overweight.

\section{INTRODUCCIÓN}

\section{4 de Mayo del 2017} Aceptado para publicación: 01 de Junio del 2017 Citar como: Rev Cient Cienc Med 2017;20(1): 16-19 comisionado, sometido a arbitraje externo.
$\mathrm{E}$ $\mathrm{n}$ la actualidad uno de los principales causantes de mortalidad a nivel mundial son los padecimientos carviovasculares 1 representando así un problema de salud pública. En un intento por aunar esfuerzos que permitan prevenir las enfermedades cardiovasculares, particularmente el Infarto Agudo de Miocardio, de manera conveniente y dar solución a problemas 
En la actualidad uno de los principales causantes de mortalidad a nivel mundial son los padecimientos carviovasculares 1 representando así un problema de salud pública. En un intento por aunar esfuerzos que permitan prevenir las enfermedades cardiovasculares, particularmente el Infarto Agudo de Miocardio, de manera conveniente y dar solución a problemas significativos de carácter multifactorial en la comunidad a nivel social, cultural, y económico se realizó el estudio Caracterización de los Factores de Riesgo Cardiovascular para Infarto Agudo de Miocardio en una población de raza garífuna, entre los 35-85 años de edad en la comunidad de Ciriboya, Colon.

Al lo largo del tiempo se ha destacado. mediante estudios observacionales y epidemiológicos los diversos factores de riesgo que predisponen al desarrollo de los padecimientos cardiovasculares que cualquier persona podría presemtarpresentar siendo los factores de riesgo generalmente potenciados entre sí y con frecuencia asociados2, debido a esto la mayor parte de las enfermedades cardiovasculares pueden ser prevenidas si se reducen estos factores de riesgo, es por esto que han sido clasificados en función a la capacidad de modificarse o no a través de la terapéutica o cambios en el estilo de vida. En Honduras, se realizorealizó un estudio de tamizaje en una población de la etnia Lenca, donde se concluyoconcluyó que los principales factores de riesgo para el desarrollo de Enfermedad Cardiovascular fueron el tabaquismo y alcoholismo.3En un estudio realizado en La Ceiba, Atlántida, Honduras se estudiaron factores de riesgo como diabetes, hipertensión arterial y obesidad entre un grupo de afrodescendientes encontrandoseencontrándose que el 18.9\% fueron diabéticos; $46.4 \%$ obesos; $46.6 \%$ hipertensos.4En el Análisis de la Situación de Salud de la Comunidad de Ciriboya se ha documentado que entre los principales factores de riesgo se encuentran el Alcoholismo con el $45.4 \%$, el Tabaquismo representando el 36.4\%, y se observa que existe un número significante de pacientes obesos teniendo en este grupo al $18.2 \%$ además de otras enfermedades de gran prevalencia en la población.

Teniendo como objetivo caracterizar los factores de riesgo cardiovascular para infarto agudo de miocardio en una población de raza garífuna, se ha tomado en cuenta los factores de riesgo no modificables como ser edad, sexo
, antecedentes familiares de enfermedad cardiovascular, diabetes mellitus e hipertensión arterial; y factores de riesgo modificables como ser obesidad, alcoholismo, tabaquismo y sedentarismo

Este estudio espera ser el primero de varios peldaños en la búsqueda y conformación de un sistema de salud inteligente y dinámico local, espera proveer las herramientas técnicas y conceptuales para modificar en forma positiva estilos de vida que contribuyan al desarrollo del Infarto Agudo al Miocardio disminuyendo así la incidencia y mejorando la calidad de vida de la población Garífuna, que representa alrededor del 3.8\% de la población hondureña y que tradicionalmente ha estado olvidada por el sistema de salud Hondureño5.

\section{MATERIALES Y MÉTODOS}

Se realizó una investigación de tipo cuantitativa descriptivo transversal

en la comunidad de Ciriboya, municipio de Iriona departamento de Colón, durante el més de enero de 2015.

La población de estudio consistióe de en 217 personas hombres y mujeres entre las edades de 35 a 85 años residentes en la comunidad de Ciriboya (ver Figura 1), según datos de Análisis Situacional de Salud de la Comunidad de Ciriboya, Iriona, Colón, elaborado por personal del Hospital Popular Garífuna de Honduras durante el año 2013. Se tomó como muestra 139 pobladores, con un nivel de confianza del 95\% y error del $5 \%$ se realizó un muestreo al azar con las personas que se encontraban en su residencia al momento del estudi o. Se excluyeron pobladores que no fueran de la raza garífuna y pobladores con discapacidad sensorial, cognitiva o motora que no pudieran colaborar en la entrevista.

Para la recolección de datos se utilizó como instrumento una encuesta realizada por la Colegio Colombiano de Cardiología y Cirugía Cardiovascular, la cual toma en cuenta factores de riesgo modificables y no modificables, y les asigna un puntaje de acuerdo a la magnitud de daño cardiovascular que pueden causar.

Se validó el instrumento el 9 de Enero de 2015 con individuos residentes del sector de las Uvas de la comunidad de Ciriboya, y no se encontraron problemas en la comprensión, forma de llenado y procesamiento del instrumento.

Se tomaron como variables: 
- Datos generales: nombre, escolaridad, teléfono y dirección; con el objetivo de brindar los resultados al Hospital Popular Garífuna para realizar campañas de intervención dirigidas.

- Riesgo cardiovascular: se determinó que el poblador tenía riesgo cardiovascular para infarto agudo de miocardio al tener una puntuación mayor de 8 de acuerdo a los puntajes establecidos para cada factor de riesgo en el instrumento.

- Edad: en los hombres se le asignó 1 punto al riego cardiovascular al grupo etario entre 35 39 años, 2 puntos al grupo entre 40 - 48 años, 3 puntos al grupo entre 49 - 53 años y 4 puntos a los de 53 años o más. En las mujeres, se le asignó 1 punto al grupo etario entre 42 - 44 años, 2 puntos al grupo entre 45 - 54 años, 3 puntos al grupo entre 55 - 73 años, 4 puntos a las de 74 años o más y 4 a aquellas que se encontraban en la menopausia sin reemplazo hormonal.

- Índice de masa corporal: se cuantificó la altura y el peso de cada poblador obteniendo el índice de masa corporal, se le asignó 1 punto a los pobladores con 25 - 27 de índice de masa corporal, 2 puntos a los pobladores con más de 27 de índice de masa corporal, se le sumó 2 puntos a aquellos pobladores con índice de masa corporal mayor a 27 con obesidad central, es decir con perímetro abdominal mayor o igual a 102 centrímetros en los hombres y mayor o igual a 88 en las mujeres6.

- Estilo de vida: se evaluó tabaquismo actual con 4 puntos y alcoholismo actual con 2 puntos.

- Actividad física y mental: se evaluó sedentarismo con 4 puntos y estrés con 4 puntos.

- Antecedentes familiares: se evaluó antecedente familiar de hipertensión arterial con 1 punto, antecedente familiar de diabetes mellitus con 2 puntos, enfermedad coronaria precoz con 4 puntos.

- Patologías específicas: se evaluó diagnóstico de hipertensión con 4 puntos, diabetes mellitus con 4 puntos y dislipidemia con 4 puntos.

Se procedió a realizar la encuesta casa por casa obteniendo las medidas necesarias ,; peso, talla y perímetro abdominal; y los datos correspondientes de todos los miembros del hogar que estaban en el rango etario adecuado

Para el procesamiento y análisis de datos,: se determinaron distribuciones porcentuales de las variables estudiadas y se clasificaron según sexo, además se cuantifico el riesgo cardiovascular de acuerdo a los puntajes anteriormente mencionados. El análisis estadístico se llevó a cabo con los programas de Epi Info 7 y Microsoft Excel.

8.- Consideraciones Éticas:Tomando en consideración los principios de justicia, beneficencia y autonomía se leyó a todos los participantes el documento de consentimiento informado donde se detallan los alcances del estudio y sus objetivos, de esta manera ellos podrán aceptar o rechazar su participación en el mismo, además de ser o no informados de los resultados y poder suspender su participación en cualquier momento.

\section{RESULTADOS}

El $64,31 \%$ de los participantes son mujeres $(n=88)$ y el $39,69 \%$ varones $(n=51)$, de los cuales $8,63 \%$ no tienen ningún grado de escolaridad, cursado primaria incompleta $31,65 \%$, primaria completa $41,73 \%$, secundaria incompleta $6,47 \%$ y secundaria completa $11,51 \%$, ninguno de los participantes había cursado educación superior. Se presentó riesgo cardiovascular en el $66.91 \%$ de los pobladores la distribución fue la siguiente: en las mujeres el $64,77 \%$ presentó riesgo cardiovascular para infarto agudo de miocardio, mientras que en los varonesel 70,59\% presentó dicho riesgo. (ver Tabla No.1).

Se estudiaron factores de riesgo modificables: alcoholismo (15,83\%), estrés (27,34\%), sedentarismo (12,95\%), tabaquismo (11,51\%), sobrepeso (54,68\%); y factores de riesgo no modificables: diabetes (8,63\%), dislipidemias (17,99\%), hipertensión arterial (32,37\%), antecedente familiar de enfermedad coronaria pre$\operatorname{coz}(12,95 \%)$, antecedente familiar de diabetes $(35,25 \%)$, antecedente familiar de hipertensión arterial (43,17\%).

\section{DISCUSIÓN}

En el estudio, se caracterizaron los factores de riesgo cardiovascular para el aparecimiento asociados ade infarto agudo de miocardio en la población Garífuna, se encontró que 93 de los 139 individuos participantes; poseen riesgo cardiovascular elevado. El 54,68\% de la población presento un índice de masa corporal mayor o igual a 25 categorizando a dicha población con sobrepeso; fundamentado en varios factores, entre ellos la actividad física diaria, pues las principales actividades son los oficios domésticos y la pesca. 
En cuanto a la prevalencia de otras enfermedades asociadas riesgo; diabetes mellitus, hipertensión arterial y dislipidemias, se encontró hipertensión arterial en $32.37 \%$ de la población en estudio. Además del $32.37 \%$ de pobladores diagnosticados, el $43,17 \%$ presentó antecedente familiar de hipertensión arterial. En estudios realizados en poblaciones hondureñas, la prevalencia de hipertensión es de $46.6 \%$ en una población de afro-hondureños en La Ceiba4, $45.52 \%$ en El Progreso7, 37,2\% en Tegucigalpa 8 y $3,3 \%$ en una población indígena de Intibucá3; hay que tomar en cuenta que en el estudio realizado solamente se tomó como hipertensos aquellos pobladores con diagnóstico de hipertensión, no se hizo estudio sobre pobladores con hipertensión arterial. La prevalencia de Diabetes Mellitus fue de $8,63 \%$, cifra que fue menor a la encontrada en una población de afrodescendientes de La Ceiba, 18.9\%.4, sin embargo al igual que la hipertensión arterial, se tomó en cuenta pobladores ya diagnosticados,

\section{REFERENCIAS}

1. González JA, González JM. Factores de riesgo para ta ocurrencia de infarto agudo de miocardio en pacientes fumadores. Revista Cubana de Salud Pública [Internet]. 2013 [citado 17 Jun 2015]; 39(4): 679- 688. Disponible en: http:// www.redalyc.org/articulo.oa? id $=21429933006$

2. Estrada L, Gómez J, Mejía A, Miranda A, Segovia A, Castaño J. Caracterización de los factores de riesgo cardiovascular para infarto agudo de miocardio en una población entre 40-80 años en el sector de la Galería de Manizales, Calda. U. Manizales [Internet]. 2003 [citado 17 Jun 2015]; 8:215. Disponible: http://ridum.umanizales.edu.co:8080/xm/ui/ bitstream/handle/6789/1453/articulo\%20factores\%20de\%20 riesgo\%20cardiovascular\%20en\%20galerias\%20en\%20columnas. $p d f$ ? sequence $=1 \&$ is Allowed $=y$

3. Reyes-García SZ, Zambrano LI, Fuentes I, Sierra M, Urquia-Osorio H. Estudio descriptivo de factores de riesgo cardiovascular a una muestra de la población de una comunidad indígena de Honduras. CIMEL [Internet]. 2011; 14(1):32-37. Disponible en : http://www.cimel.felsocem.net/ index.php/CIMEL/article/viewFile/181/137

4. Hernández A. Pria DC [Dir] Las desigualdades sociales en diabetes mellitus 2, hipertensión arterial y obesidad en las poblaciones afrohondureña y mestiza de La Ceiba [tesis]. Honduras; 2013. Universidad de San Carlos de Guatemala. Junio 2015.

5. Faúndez A, Meléndez E. Pueblos afrodescendientes de Honduras. Secretaría de Desarrollo de los Pueblos Indí- no se realizó un estudio de tamizaje.

En conclusión, nuestros datos sugieren que la población se encuentra con un nivel de riesgo alto $(66,91 \%)$, los factores de riesgo cardiovascular prevalentes para infarto agudo al miocardio en la población son diversos, pero en su mayoría son factores modificables y controlables a través de medidas

instauradas en el binomio paciente-medico. Consideramos la importancia de continuar estudios como este, ya que identificar factores de

riesgo como hipertensión arterial, diabetes mellitus, obesidad, dislipidemias, tabaquismo, entre otros, sigue siendo la estrategia fundamental para prevenir las enfermedades cardiovasculares.10

AGRADECIMIENTOS

Agradecemos a la Universidad Nacional Autónoma de Honduras por el apoyo logístico bridado para poder realizar el trabajo de campo; a la Alcaldía de Iriona, quien brindó el hospedaje en la comunidad para los investigadores y especialmente a los pobladores de Ciriboya quienes colaboraron en este estudio.

genas y Afrohondureños CMdA, Ed., Proceedings of the meeting held at La Ceiba, Honduras. Hemoglobin [Internet]. 2011; 39:1-52. Disponible en: http://tandfonline.com/ doi/abs/10.3109/03630269.2015.1012294?scroll=top\&needAccess=true\&journalCode=ihem 20

6. Fauci AS, Kasper DL, Braunwald E, Hauser SL, Longo DL, Jameson JL, et al. Harrison, Principios de Medicina Interna. Vol 2 [Libro]. 18va ed. New York: McGraw Hill; 2012. p. 1992.

7. Hall J, Hall J, Alvarenga M, Gómez O. Prevalencia de hipertensión arterial en adultos de EI Progreso. Rev Med Hond [Internet]. 2005; 73 (2): 60-64. Disponible en: http://cidbimena.desastres.hn/RMH/pdf/2005/pdf/Vol73-2-2005-2.pdf

8. Cerrato Hernández KP, Zambrano LI. Prevalencia de Hipertensión Arterial en una Comunidad Urbana de Honduras. Rev. Fac. Cienc. Méd [Internet]. 2009; 6(2):34-39. Disponible en: http://cidbimena.desastres.hn/RFCM/pdf/2009/ pdf/RFCMVol6-2-2009-6.pdf

9. Erazo BM, Ramírez GA, Cerrato LE, Pinto LJ, Castro EJ, Yanez NJ, et al. Prevalence of Hb S (HHB: c.20A>T) in a Honduran population of African descent. Hemoglobin [Internet]. 2015; 39(2):134-7. Disponible en: https://www.ncbi. nlm.nih.gov/pubmed/25707678

10. The World Health Report 2002: Reducing risks, Promoting Healthy Life. Geneva, WorldHealthOrganization. 2002.

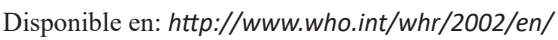

